José-Artur Paiva

\title{
Adding risk factors for potentially resistant pathogens, increasing antibiotic pressure and risk creating the "untreatable bacteria": time to change direction
}

Received: 19 December 2012

Accepted: 20 December 2012

Published online: 29 January 2013

(C) Springer-Verlag Berlin Heidelberg and ESICM 2013

This editorial refers to the article available at: doi:10.1007/s00134-012-2808-5.

J.-A. Paiva (楩)

Emergency and Intensive Care Department,

Centro Hospitalar S. João EPE, Faculdade de Medicina,

University of Porto, Porto, Portugal

e-mail: jarturpaiva@gmail.com

Trouillet et al.'s [1] landmark paper proposed a simple strategy for selecting the initial therapy of patients suspected of having ventilation-associated pneumonia (VAP). The findings of that study indicated that the duration of mechanical ventilation ( $\geq 7$ days) and the recent use of antibiotics are the two key factors favouring potentially resistant microorganisms as causative agents for ICU-acquired pneumonia.

Later, the 2005 American Thoracic Society (ATS)/ Infectious Diseases Society of America (IDSA) guidelines for the management of adults with hospital-acquired, ventilator-associated and health-care-associated pneumonia [2] added other risk factors for multidrug resistant pathogens, namely hospitalization for 2 days or more in the preceding 90 days, residence in a nursing home or extended care facility and immunosuppressive therapy or disease. This guideline also stated that a high frequency of antibiotic resistance in the community or in the specific hospital unit predicted the occurrence of these pathogens as causative agents.

Martin-Loeches et al. [3] confirm the importance of taking into consideration the environment to select empiric antibiotic therapy for hospital-acquired pneumonia
(HAP)/VAP and add another independent risk factor for potentially resistant microorganisms as causative pathogen-the development of severe sepsis or septic shock associated with the HAP/VAP episode.

The consideration of all these risk factors-late onset, recent use of antibiotics, hospitalization for 2 days or more in the preceding 90 days, residence in a nursing home or extended care facility, immunosuppressive therapy or disease, high prevalence of potentially resistant pathogens and development of severe sepsis or septic shock-leads to a very frequent use of empiric broadspectrum antibiotics to guarantee coverage of potentially resistant pathogens, namely methicillin-resistant Staphylococcus aureus, Pseudomonas aeruginosa and Acinetobacter spp. This maximizes antibiotic appropriateness and improves outcome [4], but it also markedly increases antibiotic consumption and antibiotic pressure, leading to the selection and emergence of antimicrobial resistance.

Therefore, a vicious circle of higher antibiotic pressure and higher prevalence of antimicrobial resistance is generated. In many health-care settings, multidrug resistance in gram-negative bacillary infections has severely restricted therapeutic options and situations were reported in which no effective antibiotics were available to treat life-threatening infections [5]. In fact, not only are $P$. aeruginosa and Acinetobacter spp. increasingly resistant to ceftazidime, fluoroquinolones and carbapenems, but Klebsiella pneumoniae and Escherichia coli are also more often resistant to carbapenems by harbouring carbapenemases or nucleoside diphosphate enzymes [6]. It is mandatory to preserve the antibiotic and protect this precious resource that has helped us decrease infectious and global mortality in the last 60 years [7].

For that purpose, we have to reflect on the path we have been taking, as adding new risk factors for potentially resistant bacteria as a VAP causative agent is a risky step that must be taken cautiously. 
In fact, a few issues should be discussed and analysed:

1. How strong is the evidence associated with the risk factors put forward by Martin-Loeches et al. [3]? Concerning high prevalence of potentially resistant bacteria, it is logical that an event or fact predicts its own occurrence later, but reasons for the association between severity of VAP and the resistance of the pathogen are less clear and less self-evident. In fact, as the authors state, a recent study [8] showed the opposite: that the severity of VAP relates to clinical status prior to VAP, namely preVAP SOFA, but not to the type of causative bacteria and that the occurrence of new organ dysfunction during VAP was similar regardless of the pathogen. Therefore, in our opinion, further studies and evidence are needed to accept this risk factor as a reason to broaden empiric antibiotic coverage.

2. Do we have the conditions that allow us to broaden the initial empiric regimen without significantly increasing antibiotic pressure and inducing the emergence of antimicrobial resistance? De-escalation and antibiotic stewardship are the two strategies rightly suggested to avoid the vicious circle of broader initial antibiotic therapy and higher level and prevalence of antimicrobial resistance. A de-escalation strategy aimed at controlling antibiotic prescription and subsequently minimizing the emergence of resistance should be part of antimicrobial stewardship in clinical practice [9]. Available data suggests that the rate of de-escalation can be increased and this could result in a potential benefit in antibiotic consumption. In fact, the rate of deescalation has varied in published studies from as low as $22 \%$ to as high as $74 \%$ [10]. Fear of changing a "winning hand" and overcautious interpretation of negative culture results are two of the reasons for this low frequency of de-escalation. Even when it is performed, it often occurs later than the third day of antibiotic treatment, as microbiological results are available later than expected and clinical response is often slow. The strategy would be much more effective for the prevention of antibiotic resistance if faster cultural or non-cultural microbiological methods were reliable and used. These technological advances, such as matrix assisted laser desorption ionization time-offlight mass spectrometry, technologies based on nucleic acid extraction from positive blood cultures or multiplex real-time polymerase chain reaction [11, 12], are seldom used in clinical practice. On the other hand, antibiotic stewardship strategies are still inexistent in most hospitals. Therefore, broad-spectrum initial empiric antibiotic therapy often lingers for too many days and sometimes for the whole duration of therapy. 3. Can we not reduce the incidence of potentially resistant microorganisms as causative agents of early-onset VAP by infection control strategies? Especially in centres with a high prevalence of these pathogens, many of these early-onset VAPs are exogenous in terms of acquisition and the institution of infection control measures, such as better hand hygiene and cleaning of surfaces, would decrease this rate and make broad-spectrum antibiotic therapy less needed. Therefore, adding spectrum or number of initial antibiotics is not the only and not even the clever answer; adding infection control is certainly a way to increase antibiotic appropriateness without promoting antibiotic pressure.

4. Are we not overtreating late-onset or early-onset with ATS/IDSA risk factors VAP (group 2) as much as we are undertreating early-onset without risk factors VAP (group 1)? The study by Martin-Loeches et al. [3] shows that the frequency of potentially resistant microorganisms was similar in group 1 and in group 2 patients, respectively 50.7 and $59.8 \%$. This clearly means that using the ATS/IDSA guidelines we are undertreating some of the group 1 VAP, but we are equally overtreating some of the group 2 VAP. It will be equally important to solve both inadequacies. Therefore, more than adding new risk factors to the previously known, we actually need better discriminating factors. Community-acquired pneumonia, health-care-associated pneumonia, early-onset HAP/ VAP, late-onset HAP/VAP and end-of-life pneumonia are a continuum in which the frequency of multidrug resistant pathogens increases progressively from the first to the last one, never being zero or $100 \%$. In this unified concept of pneumonia, risk assessment for potentially resistant bacteria should always be done and in an individual patient-centred way.

In conclusion, by adding risk factors for potentially resistant microorganisms we are promoting antibiotic pressure by increasing the spectrum of initial empiric therapy which often lingers for too many days as microbiological results, clinical response assessment and antimicrobial stewardship occur later than ideally. Unless we improve these conditions, bringing new tools and methodologies to the clinical setting, and find better discriminating factors we risk creating the "untreatable bacteria". 


\section{References}

1. Trouillet JL, Chastre J, Vuagnat A, Joly-Guillou ML, Combaux D,

Dombret MC, Gibert C (1998)

Ventilator-associated pneumonia caused by potentially drug resistant bacteria. Am J Respir Crit Care Med 157:531-539

2. ATS/IDSA (2005) Guidelines for the management of adults with hospitalacquired, ventilator-associated and health care associated pneumonia. Am J Respir Crit Care Med 171:388-416

3. Martin-Loeches I, Deja M, Koulenti D, Dimopoulos G, Marsh B, Torres A, Niederman M, Rello J (2013)

Potentially resistant microorganisms in intubated patients with hospitalacquired pneumonia: the interaction of ecology, shock and risk. Intensive Care Med. doi:10.1007/s00134-012-2808-5

4. Kollef KE, Schramm GE, Wills AR, Reichley RM, Micek ST, Kollef MH (2008) Predictors of 30-day mortality and hospital costs in patient with ventilator-associated pneumonia attributed to potentially antibioticresistant gram-negative bacteria. Chest 134:1208-1216
5. Koo SH, Kwon KC, Cho HH, Sung JY (2010) Genetic basis of multi-resistant Acinetobacter baumannii clinical isolates from three university hospitals in Chungcheong Province, Korea. Korean J Lab Med 30:498-506

6. Kumarasamy KK, Toleman MA, Walsh TR et al (2010) Emergence of a new antibiotic resistance mechanism in India, Pakistan and the UK: a molecular, biological and epidemiological study. Lancet Infect Dis 10:597-602

7. Carlet J, Collignon P, Goldmann D, Goossens H, Gyssens IC, Harbarth S, Jarlier V, Levy SB, N'Doye B, Pittet D, Richtmann R, Seta WH, van der Meer JWM, Voss A (2011) Society's failure to protect a precious resource: antibiotics. Lancet 378:369-371

8. Damas P, Layios N, Seidel L, Nys M, Melin P, Ledoux D (2011) Severity of ICU-acquired pneumonia according to infectious microorganisms. Intensive Care Med 37:1128-1135
9. Dellit T, Owens R, McGowan J et al (2007) Infectious Diseases Society of America and the Society for Healthcare Epidemiology of America guidelines for developing an institutional program to enhance antimicrobial stewardship. Clin Infect Dis 44:159-177

10. Niederman MS, Soulountsi V (2011) De-escalation therapy: is it valuable for the management of ventilatorassociated pneumonia? Clin Chest Med 32:517-534

11. Leggieri N, Rida A, François $P$, Schrenzel J (2010) Molecular diagnosis of bloodstream infections: planning to (physically) reach the bedside. Curr Opin Infect Dis 23:311-319

12. Pletz MW, Wellinghausen $\mathrm{N}$, Welte $\mathrm{T}$ (2011) Will polymerase chain reaction (PCR)-based diagnostics improve outcome in septic patients? A clinical view. Intensive Care Med 37:1069-1076 